\title{
PUBLICACIÓN CIENTÍFICA DE ASESORES DE TESIS DE PSICOLOGÍA DE 30 UNIVERSIDADES PERUANAS
}

\author{
Oscar Mamani-Benito $^{\text {a }}{ }^{10}$, Araseli Verastegui-Diaz ${ }^{b}{ }^{\mathbb{D}}$, Christian $\mathbf{R}$. \\ Mejia $^{\mathrm{C}}$, \& Tomás Caycho-Rodríguez ${ }^{\mathrm{d}}(\mathbb{D})$
}

Universidad Peruana Unión, Juliaca, Perú. a

Universidad Ricardo Palma, Lima, Perú.

Universidad Continental, Lima, Perú. ${ }^{c}$

Universidad Privada del Norte, Lima, Perú. ${ }^{\mathrm{d}}$

\begin{abstract}
RESUMEN
El objetivo fue determinar la frecuencia de publicación científica de 231 asesores de tesis de psicología de 30 universidades peruanas, mediante un estudio descriptivo-transversal. Los resultados revelan que 58.4\% nunca ha publicado un artículo científico; solo un $26.8 \%$ lo ha hecho en los últimos tres años y un $18.2 \%$ en los últimos cinco años. Un $23.8 \%$ ha logrado publicar en revistas indexadas en SciELO y $14.7 \%$ en Scopus. En 10 de las 30 universidades más del 50\% de los docentes había publicado algún artículo científico y solo de 2 a 5 universidades tuvieron docentes que han publicado en los últimos 3 y 5 años. Se concluye que la producción científica de los asesores de tesis en psicología en el Perú es baja. Esto puede repercutir en la generación de conocimientos validados por la comunidad científica y, por ende, en la perdida de oportunidades para el desarrollo de una psicología peruana basada en evidencia científica que atienda las necesidades del país.
\end{abstract}

Palabras Claves

tesis académicas; investigación; docentes; Perú

\begin{abstract}
The objective was to determine the frequency of scientific publication of 231 psychology thesis advisors from 30 Peruvian universities, through a cross-sectional study. The results reveal that $58,4 \%$ have never published a scientific article; Only $26.8 \%$ have done so in the last three years and $18.2 \%$ in the last five years. $23.8 \%$ have managed to publish in journals indexed in SciELO and 14.7\% in Scopus. In 10 of the 30 universities, more than $50 \%$ of the teachers had published a scientific article and only 2 to 5 universities had teachers who have published in the last 3 and 5 years. It is concluded that the scientific production of thesis advisors in psychology in Peru is low. This can have an impact on the generation of knowledge validated by the scientific community and, therefore, on the loss of opportunities for the development of a Peruvian psychology based on scientific evidence that meet the needs of the country.
\end{abstract}

Keywords

academic dissertations; research; professors; Peru

\footnotetext{
${ }^{1}$ Correspondence about this article should be addressed to Oscar Mamani-Benito: oscar.mb@upeu.edu.pe
} 


\section{SCIENTIFIC PUBLICATION OF PSYCHOLOGY THESIS ADVISORS FROM 30 PERUVIAN UNIVERSITIES}

\section{Introducción}

La investigación es considerada esencial para el crecimiento económico y social a largo plazo (Franco y Pinho, 2019). En este contexto, la universidad como institución de educación superior y de investigación (Arriola-Quiroz, Curioso, Cruz-Encarnacion y Gayoso, 2010), tiene como objetivo principal la producción científica y la transmisión del conocimiento (Berbegal-Mirabent, Sánchez y Ribeiro-Soriano, 2015). Sin embargo, a pesar de que en América Latina se observa un incremento del número de científicos e instituciones dedicados a la investigación, las brechas entre países desarrollados y latinoamericanos son alarmantes debido a que estos no consideran a la investigación como una prioridad y asignan presupuestos que, comparados con los países desarrollados, donde la investigación se ha convertido en una industria más, resultan risibles; por consiguiente la diferencia entre unos y otros responde a políticas de estado (Ciocca y Delgado, 2017). Los países latinoamericanos aún no brindan la importancia debida al desarrollo científico y tecnológico, lo que se evidencia en que a pesar del incremento notable de la producción latinoamericana esta representa en la actualidad solo el 5\% de las publicaciones mundiales, comparadas con Europa (30\%) y Norteamérica (30\%) (Cepeda, Pazmiño y Medrano, 2018), además al evaluar la contribución regional por autores, los autores latinos representamos menos del 5.2\% en comparación con el $88 \%$ de los autores norteamericanos (Pérez-Batres, Pisani y Doh, 2010).

Esta realidad no es ajena al Perú donde se observa una limitada producción científica que aumentó de 121 artículos en el 2000 a 1210 en 2009, sin embargo, este incremento sigue siendo insuficiente (Huamaní y Mayta-Tristán, 2010; Yagui, Espinoza, Caballero, Castilla, Garro yamaguchi, Mayta-Tristán, Velásquez y Cabezas, 2010). Una de las soluciones a este problema, es la promulgación de la ley universitaria $N^{0} 30220$, que obliga a los estudiantes a preparar, sustentar y aprobar una investigación (tesis) para el logro del título profesional (Alarcon-Ruiz y Quezada, 2018). En el marco de esta ley, la tesis es considerada un vehículo que permite el fortalecimiento de la investigación en el contexto universitario (León, 2016) y el primer acercamiento de los estudiantes a la investigación científica (Castro, Cósar-Quiroz, Arredondo-Sierralta y Sihuay-Torres, 2018). 
La tesis es un trabajo académico de elevado rigor científico, cuyo proceso de elaboración permite el desarrollo de conocimientos y habilidades básicas para la investigación científica, el pensamiento crítico y la solución de problemas (Obuku, Lavis, Kinengyere, Ssenono, Ocan, Mafigiri, Ssengooba, Karamagi y Sewankambo, 2018). Desafortunadamente, la tesis convencional, rara vez, y no obligatoriamente, deriva en un trabajo publicado (Wilson, 2002). Es más, generalmente se la considera una mera formalidad o incluso una carga tanto para los estudiantes como para los profesores (Shukla, Tripathi, \& Devi, 2019). Hallazgos recientes indican que solo el 25\% de las tesis se publican más tarde en revistas científicas que cuenten con revisión por pares (Evans, Amaro, Herbert, Blossom, \& Roberts, 2018). En el Perú, cerca del 17.6\% de las tesis con un formato tradicional en una universidad privada lograron ser publicadas, lo cual aumentó a un $22.4 \%$ cuando el formato de presentación cambió al de un artículo científico, llegando al $40.3 \%$ cuando la tesis, en este nuevo formato, se envió a revisión en una revista indizada (Mayta-Tristán, 2016). En este contexto, algunos sugieren que el proceso de tesis culmine con la difusión de los resultados de la investigación a la comunidad científica, principalmente por medio de la publicación en una revista indizada (Atamari-Anahui, Roque-Roque, Robles-Mendoza, Nina-Moreno y Falcón-Huancahuiri, 2015; Dhaliwal, Singh y Bhatia, 2010; Taype-Rondán, Carbajal-Castro y ArrunateguiSalas, 2012); esto, en función de que la producción científica puede plasmarse en artículos publicados en revistas especializadas e indizadas, revistas no indizadas y tesis (Pamo Reyna, 2005), donde solo el primero permite que el trabajo de investigación tenga impacto internacional (Rodríguez, et al.2018). Además, las publicaciones científicas permiten medir objetivamente la productividad de los investigadores (Godoy, Navarro, \& Escoto, 2008). De este proceso, resulta importante el rol del asesor de la tesis que, debido a su mayor experiencia en un campo específico de la ciencia, debe orientar y acompañar al estudiante (tesista) a lo largo de toda la investigación, desde la concepción de la idea de investigación, la solución del problema, hasta la redacción del informe científico a sustentar (Alarcon-Ruiz y Quezada, 2018; Llaurado-Serra et al., 2018; Nordsteien, Horntvedt y Syse, 2017). Aun así, se sugiere que el trabajo del asesor no culmina con la sustentación, sino con la publicación de la tesis en una revista científica; esto debido a que la finalidad de realizar una tesis es generar y compartir conocimiento (Castro, Cósar-Quiroz, Arredondo-Sierralta y Sihuay-Torres, 2018; Pasarica, Bailey y Cendán, 2019). La evidencia indica que la experiencia previa de publicación de un asesor (entre dos o más artículos publicados anualmente) triplica la probabilidad de que el 
estudiante culmine sus tesis (Oyola-García, 2015). Si bien no hay evidencia objetiva previa, los antecedentes en publicación de los asesores, podrían orientar la publicación de las tesis en revistas científicas, lo que podría redundar en una mejor producción de conocimiento relevante para el país. Sin embargo, reportes previos han mostrado que gran cantidad de los asesores de tesis no siempre cumplen con la expectativa de los asesorados y muchos incluso pueden ser una traba en el proceso (Mayta-Tristán, 2016), además de no tener experiencia en publicación científica (Castro Rodríguez, Sihuay-Torres y PerezJiménez, 2018). Esto ha originado que los resultados de las tesis no lleguen a publicarse y queden solo en los archivos de la universidad (Castro-Rodríguez, 2018; Castro, CósarQuiroz, Arredondo-Sierralta y Sihuay-Torres, 2018; Zafra-Tanaka y Castillo, 2016).

La experiencia en investigación de los asesores de tesis expresada en su productividad científica, ha sido recientemente estudiada en el contexto peruano. Una investigación (Atamari-Anahui, Sucasaca-Rodríguez, Cruz y Jhonatan, 2016), señaló que el $47.1 \%$ de 68 asesores de tesis de pregrado de una escuela de medicina del Cusco publicó un artículo (sea del tipo que sea) en su vida, mientras que el 39.7\% publicó en alguna oportunidad un artículo original. Respecto a artículos publicados en revistas indizadas, el $11.8 \%$ de estos asesores publicó en los 3 últimos años en revistas indizadas en Medline y Scopus; y solo el 5.9\% publicó un artículo original en este mismo periodo. Otro estudio (Alarcon-Ruiz y Quezada, 2018) reportó que el 63\% de 19 asesores de tesis de la Facultad de Medicina de una universidad privada de Lima, había publicado alguna vez en su carrera profesional algún artículo; mientras que el 53\% publicó en alguna oportunidad un artículo original. Estos porcentajes disminuyen al evaluar las publicaciones de artículos originales en revistas indizadas en Scopus y Medline en los últimos 5 años, a un $26 \%$ y $21 \%$ respectivamente. Por otro lado, cuando se analiza la publicación de artículos originales en los 2 últimos años en las bases de datos mencionadas, el porcentaje cae a un 5\%. Los resultados de ambos estudios expresan una baja tasa de publicación de artículos científicos originales de los asesores de tesis.

Como se observa, el tema de producción científica de los asesores de tesis se ha investigado en diversas carreras, pero aún no se ha reportado un análisis de la situación de la carrera de psicología. En este sentido, el objetivo de la investigación fue determinar la frecuencia de publicación científica de los asesores de tesis de pregrado de 30 universidades peruanas que enseñan psicología. 


\section{Método}

\section{Diseño}

Investigación descriptiva-transversal

\section{Participantes}

Se trabajó con una población de 231 docentes que asesoraron tesis de pregrado en psicología, las cuales fueron aprobadas en el periodo 2014 - 2017. La configuración de esta cantidad fue el resultado de una búsqueda de trabajos de grado en los repositorios digitales de 55 universidades peruanas que según el portal Ponte en Carrera del MINEDU (2015) ofertan la formación del psicólogo(a). De este grupo se halló que solo 30 universidades mantenían trabajos de tesis en archivo de tipo extenso, mientras que en el grupo restante (25) se evidenció que aún no cuentan con un portal de almacenamiento o no se tiene la autorización del autor para la visualización completa o descarga de la tesis.

Una vez identificadas las universidades con repositorio digital, se inició una búsqueda de archivos de tesis de pregrado con opción a visualización o descarga completa. Aquí se logró recolectar 1191 trabajos, en los cuales se identificó a los asesores recurriendo a la portada principal o en casos especiales a la página de las firmas del jurado evaluador.

\section{Variables $e$ instrumento}

La variable principal fue la publicación científica por parte de los asesores de tesis, para lo cual, se recopiló si publicaron alguna vez en su vida, en los últimos 3 o 5 años; además, si es que publicó en la base de datos Scopus o SciELO. Cada una de estas variables se tomó en su forma categórica: Si o No.

Las variables que describirían a cada asesor fueron: el sexo (masculino o femenino), el grado académico máximo que tenían (titulado, magíster o doctor), el vínculo laboral (si laboraba a tiempo parcial o completo), el tipo de institución donde labora (estatal o privada) y la universidad en específico (cada una de las 30 universidades que tenían repositorio de tesis). Se diseñó una lista de cotejo para la captura de todas estas características demográficas y de producción científica de los asesores. 


\section{Procedimiento}

Se identificaron los nombres y apellidos de cada asesor(a), se realizó una exploración con sus datos en el Directorio Nacional de Investigadores (DINA), actualmente CTI VITAEque gestiona el Consejo Nacional de Ciencia, Tecnología e Innovación (CONCYTEC); así mismo, se recurrió al Registro de Grados y Títulos de la Superintendencia Nacional de Educación Superior Universitaria (SUNEDU). De ambos portales se pudo recoger información respecto al grado académico, filiación, vínculo laboral y producción científica consignada por los mismos docentes; no obstante, para corroborar este último dato, se realizó una búsqueda consignando los apellidos de los autores en el motor de búsqueda de Google Académico y la base de datos de Scopus, el cual logró corroborar una cantidad real y exacta de publicaciones en distintas revistas indexadas de la especialidad.

En todo momento se respetó la ética en la investigación, la información de los repositorios es pública, al igual que la que se consultó en DINA, CONCYTEC, la SUNEDU y en los buscadores de los trabajos científicos publicados. No se brindan resultados individuales o de algún asesor en particular, además, una vez elaborada la base se anonimizó para respetar la privacidad.

\section{Análisis de datos}

Una vez obtenida la información se generó una base de datos en el programa Microsoft Excel, con dicho programa se pudo realizar la estadística descriptiva, en donde se obtuvo las frecuencias y porcentajes de todas las variables. Con estos resultados se pudo generar las tablas científicas.

\section{Resultados}

De los 231 asesores evaluados, existe mayor participación de docentes mujeres (50.2\%), por otra parte, se observa que el $39.8 \%$ de docentes ostentan el grado de magister/maestro, en cambio, el $35.5 \%$ solo evidencia título profesional y en menor proporción el grado de doctor $(24.7 \%)$. Un $64.9 \%$ se vincula a tiempo completo con la universidad donde labora. Finalmente, el $83.1 \%$ labora en universidades privadas.

Respecto a la producción científica de los asesores, el $58.4 \%$ no ha logrado publicar alguna vez en su vida; solo un $26.8 \%$ ha publicado en los últimos tres años y un $18.2 \%$ en los últimos cinco años. De igual manera, un $23.8 \%$ ha logrado publicar en 
revistas indexadas en SciELO y en menor proporción Scopus (14.7\%).

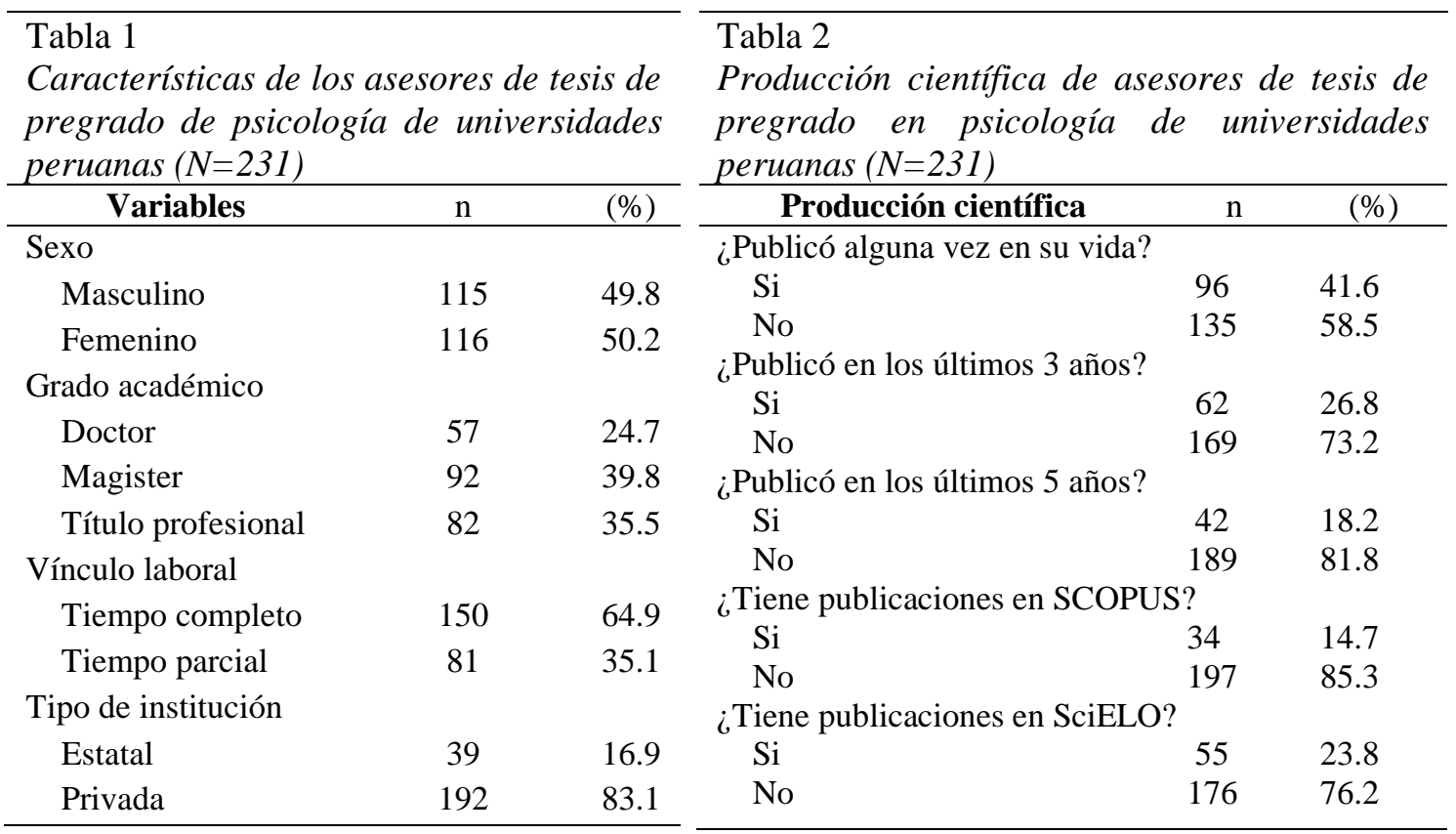

La tabla 3 muestra con precisión las contribuciones científicas de los asesores de tesis según universidad de procedencia. En base a la proporción de docentes por cada institución, se halló que en diez de las universidades (PUCP, URP, UPN, UCSM, UPC, UPCH, USIL, UNMSM, UNFV y USMP) más del 50\% de sus asesores ha logrado publicar alguna vez en su vida, en cambio, dentro de los últimos cinco años solo asesores de PUCP y UNMSM y dentro de los últimos tres años, solo asesores de PUCP, URP y UNMSM. En cuanto a publicaciones indizadas en SciELO, se observa una proporción significativa en asesores de PUCP, URP, UPC, UPCH y UNMSM, mientras que en Scopus solo asesores de PUCP y UPC. 


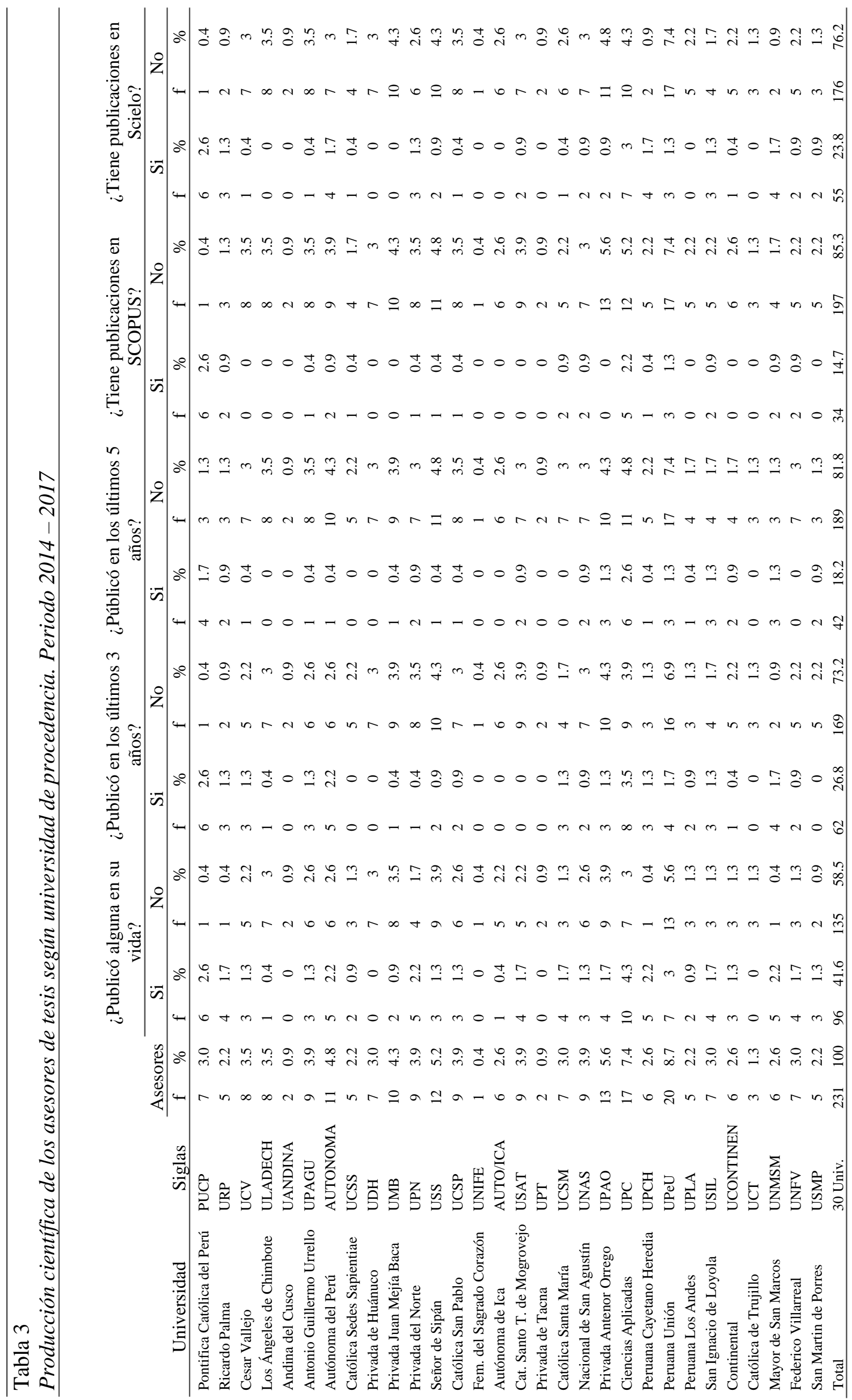




\section{Discusión}

Se tuvo como objetivo determinar la frecuencia de publicación científica de asesores de tesis de universidad peruanas que enseñan psicología. Luego del análisis respectivo se obtuvieron datos interesantes los cuales se discuten a continuación.

Seis de cada diez asesores no habían publicado en toda su vida, solo uno de cada cuatro lo han hecho en los últimos tres años y dos de cada cinco en los últimos cinco años. Estos resultados son muy similares a los reportados en una investigación donde se mostró que solo seis de cada diez docentes de investigación habían publicado alguna vez en su vida, aunque en dichos docentes de medicina los porcentajes de publicación en los últimos 3 y 5 años fueron mejor que en los que nosotros evaluamos en psicología (Pereyra-Elías, Huaccho-Rojas, Taype-Rondan, Mejia y Mayta-Tristán, 2014). Aunque las poblaciones son parecidas, no son exactamente similares ya que, la investigación en docentes médicos fue realizada en aquellos que enseñaban cursos que tenían que ver con investigación, en cambio, nuestros resultados son en los que ya son asesores de tesis -que deberían incluso publicar mucho más ya que, los docentes de investigación no siempre llegan a ser asesores-. Esto nos podría mostrar que existe aún mucha deficiencia en este grupo de asesores lo cual ha sido mostrado en diferentes grupos de profesionales de la salud (Atamari-Anahui et al., 2016), directivos que se dedican a la investigación (MaytaTristán, Pereyra-Elías y Mejía, 2013) (Pulido-Medina, Hamon-Rugeles, López-Ramírez, Quimbayo-Cifuentes y Mejía, 2017) y hasta profesionales con post grado (CorralesReyes, Reyes- Pérez y Díaz-Santoya, 2019).

Menos de la cuarta parte ha logrado publicar en revistas indexadas en SciELO y menos de la sexta parte lo ha hecho en Scopus. Estos resultados solo ratifican lo anteriormente reportado, ya que, en la investigación de Pereyra-Elías se muestra que uno de cada tres docentes si había llegado a publicar en Scopus lo que significa más del doble de lo que han publicado nuestros asesores de psicología (Pereyra-Elías et al., 2014). No se encontró publicaciones que muestren la cantidad de docentes que han publicado en SciELO, pero si encontramos que del total de artículos publicados en SciELO Perú, el $12 \%$ contaba con un estudiante de medicina, lo que nos puede mostrar que en otras carreras se ha incentivado la publicación desde la etapa estudiantil (Huaraca Hilario, Apaza Alccayhuaman y Mejia Alvarez, 2017). Es muchas veces debido a esto que los estudiantes perciben que sus docentes no están capacitados para ayudarlos a culminar sus investigaciones (Mejia, Cáceres, Vera, Inga-Berrospi y Mayta-Tristán, 2016), a pesar que 
ellos perciben que la investigación es muy importante, pero que no la saben impartir como se debería (Hamon-Rugeles D, Pulido-Medina C y López-Ramírez E, 2017).

Solo un tercio de las universidades tenían a más del 50\% de sus docentes que había publicado alguna vez en su vida. Algo similar se ha reportado en la carrera de medicina, en donde una investigación similar encontró que solo la mitad de las escuelas de investigación solicitaban un trabajo final post un curso de investigación y que solo una escuela solicitó que se realice una publicación como parte de la cátedra (Taype-Rondán, Huaccho-Rojas J, Pereyra-Elías R, Mejia CR y Mayta-Tristán P, 2015), lo que explica el bajo conocimiento que tienen los estudiantes en temas de investigación, tanto en estudiantes que cursan el pre grado, como en aquellos que ya están en el internado, siendo estos los que deberían usar la mayor cantidad de información científica de calidad (Mejia et al., 2016).

Por último, solo de 2 a 5 universidades tuvieron docentes que han publicado en los 3 y 5 últimos años, tanto en SciELO como en Scopus. Lo que nos muestra que la gran mayoría de universidades aún no cuenta con docentes/asesores que sepan realizar publicaciones científicas. Lo que también ha sido mostrado por otros investigadores quienes demuestra que no todas las facultades contaban con docentes de investigación que habían publicado en Scopus o Google Académico (Taype-Rondán et al., 2015). Otra reciente investigación ha mostrado que son pocas las facultades de medicina que han apostado por tener a docentes REGINA (Mejia y Montero, 2018), sabiendo que son estos docentes los que realmente hacen investigación. Lo que debería ser evaluado por cada institución educativa ya que, uno de los pilares de la universidad es el poder realizar investigación, pero esto parece que ha sido relegado hasta la actualidad.

En conclusión, solo seis de cada diez asesores de tesis habían publicado alguna vez en su vida, menos del $30 \%$ lo había hecho en los últimos tres y cinco años. Un tercio de las universidades evaluadas tuvieron a la mayoría de sus asesores con alguna publicación, además, menos de cinco universidades destacaron por tener docentes que han publicado en los 3 y 5 últimos años, tanto en SciELO como en Scopus.

La principal limitación que se tuvo fue el sesgo de selección ya que, al realizar un muestreo no aleatorio no se pueden extrapolar los resultados a los asesores de tesis de psicología de todo el Perú, sin embargo, los resultados que se han obtenido son realmente importantes en función de que se tomó la totalidad de datos que se encontró en los repositorios institucionales de la mayoría de las universidades peruanas que cuentan con la carrera, lo que nos permite tener una idea de lo que realmente podría estar sucediendo. 
Así también es importante tomar en cuenta el sesgo de información ya que, se perdió la oportunidad de analizar otras variables importantes al solo obtener la información que estuviese en los lugares que se consultó, por lo que, otras investigaciones deberían realizar futuros análisis con una mayor cantidad de variables relacionadas. Ante ello, surge la necesidad de replicar el estudio orientando el análisis hacia los factores asociados a la baja publicación científica de asesores de tesis, así también, limitantes tanto en las tesis como en sus autores para poder comunicar sus resultados en revistas indexadas de la especialidad. 


\section{Referencias}

Arriola-Quiroz, I., Curioso, W. H., Cruz-Encarnacion, M., \& Gayoso, O. (2010). Characteristics and publication patterns of theses from a Peruvian medical school. Health Information y Libraries Journal, 27(2), 148-154. https://doi.org/10.1111/j.1471-1842.2010.00878.x

Atamari-Anahui, N., Sucasaca-Rodríguez, C., Contreras-Sotomayor, S., Aguilar-Muñíz, A., Velásquez-Cuentas, L., \& Mejía, C. R. (2016). Factores asociados a las prácticas de publicación de médicos que laboran en hospitales de Cusco, Perú. Revista Cubana de Información en Ciencias de la Salud, 27(4), 531-544. http://www.rcics.sld.cu/index.php/acimed/article/view/949

Alarcon-Ruiz, C. A. \& Quezada, M. A. (2018). Publicación de artículos científicos por asesores de tesis de una Facultad de Medicina. Revista Médica Herediana, 29(3), 152-157. https://doi.org/10.20453/rmh.v29i3.3403

Atamari-Anahui, N., Roque-Roque, J. S., Robles-Mendoza, R. A., Nina-Moreno, P. I. \& Falcón-Huancahuiri, B. M. (2015). Publicación de tesis de pregrado en una facultad de Medicina en Cusco, Perú. Revista Médica Herediana, 26(4), 217-221. http://www.scielo.org.pe/scielo.php?script=sci_arttext\&pid=S1018130X2015000400003

Atamari-Anahui, N., Sucasaca-Rodríguez, C., Cruz, M. S. \& Jhonatan, A. (2016). Publicación científica de asesores de tesis de pregrado en una escuela de medicina de Cusco, Perú. Investigación en educación médica, 5(20), 279-280. http://dx.doi.org/10.1016/j.riem.2016.05.002

Berbegal-Mirabent, J., Sánchez, J. L., \& Ribeiro-Soriano, D. E. (2015). Universityindustry partnerships for the provision of RyD services. Journal of Business Research, 68(7), 1407-1413. https://doi.org/10.1016/j.jbusres.2015.01.023

Castro Rodríguez Y., Cósar-Quiroz, J., Arredondo-Sierralta, T., \& Sihuay-Torres, K. (2018). Producción científica de tesis sustentadas y publicadas por estudiantes de Odontología. $\quad$ Educación $\quad$ Médica, $\quad 19(2), \quad$ 85-89. https://doi.org/10.1016/j.edumed.2017.04.002

Castro Rodríguez, Y., Sihuay-Torres, K., \& Perez-Jiménez, V. (2018). Producción científica y percepción de la investigación por estudiantes de odontología. Educación Médica, 19(1), 19-22. https://doi.org/10.1016/j.edumed.2016.11.001

Castro-Rodríguez Y. (2018). Indicadores bibliométricos de las tesis sustentadas por estudiantes de Odontología, Perú. EDUMECENTRO, 10(4), 1-19. http://scielo.sld.cu/pdf/edu/v10n4/edu01418.pdf

Corrales-Reyes, I. E., Reyes- Pérez, J. J. A., \& Díaz-Santoya, M. L. A. (2019). Asesores de tesis y publicación de artículos científicos en el pregrado. Revista Médica Herediana, 29(4), 264. https://doi.org/10.20453/rmh.v29i4.3454

Ciocca, D. R., \& Delgado, G. (2017). The reality of scientific research in Latin America; an insider's perspective. Cell Stress and Chaperones, 22(6), 847-852. https://doi.org/10.1007/s12192-017-0815-8

Cépeda, K., I., Pazmiño, L. \& Medrano, E. L. (2018). Evolución de la Investigación Científica en América Latina. RECIMUNDO: Revista Científica de la Investigación $\quad y \quad$ el Conocimiento, 2(2), 464-476. http://www.recimundo.com/index.php/es/article/view/240

Dhaliwal, U., Singh, N., \& Bhatia, A. (2010). Masters theses from a university medical college: publication in indexed scientific journals. Indian journal of ophthalmology, $\quad 58(2)$,

101-104. https://www.ncbi.nlm.nih.gov/pubmed/20195030 
Evans, S. C., Amaro, C. M., Herbert, R., Blossom, J. B., \& Roberts, M. C. (2018). “Are you gonna publish that?' Peer-reviewed publication outcomes of doctoral dissertations in psychology. PLOS ONE, 13(2), e0192219. https://doi.org/10.1371/journal.pone.0192219

Franco, M., \& Pinho, C. (2019). A case study about cooperation between University Research Centres: Knowledge transfer perspective. Journal of Innovation and Knowledge, 4(1), 62-69. https://doi.org/10.1016/j.jik.2018.03.003

Godoy, M. E. R., Navarro, E., \& Escoto, A. S. D. (2008). Impacto de la producción editorial del Instituto Nacional de Psiquiatría Ramón de la Fuente, entre 1995 y 2006, de acuerdo con el ISI Web of Science. Salud mental, 31(1), 3-17. http://www.scielo.org.mx/scielo.php?script=sci_arttext\&pid=S0185$33252008000100002 \& \operatorname{lng}=$ es\&nrm=iso\&tlng=es

Huamaní, C., \& Mayta-Tristán, P. (2010). Producción científica peruana en medicina y redes de colaboración, análisis del Science Citation Index 2000-2009. Revista Peruana de Medicina Experimental y Salud Pública, 27(3), 315-325. http://www.scielo.org.pe/pdf/rins/v27n3/a03v27n3.pdf

Hamon-Rugeles D, Pulido-Medina C, Q.-C.A., \& López-Ramírez E, M. C. (2017). Percepción de la importancia, el apoyo y la realización de investigación entre los directivos y presidentes de las asociaciones científicas estudiantiles de Colombia. Ciencia e Investigación Medico Estudiantil Latinoamericana, 22(1), 21-25. https://www.cimel.felsocem.net/index.php/CIMEL/article/download/738/385

Huaraca Hilario, C. M., Apaza Alccayhuaman, A., \& Mejia Alvarez, C. (2017). Realidad peruana de la publicación científica estudiantil en los últimos diez años. Educación Médica $\quad$ Superior, $\quad 31(3), \quad 124-134$. http://www.ems.sld.cu/index.php/ems/article/view/1019

Llaurado-Serra, M., Rodríguez, E., Gallart, A., Fuster, P., Monforte-Royo, C., \& De Juan, M. Á. (2018). Assessing the competences associated with a nursing Bachelor thesis by means of rubrics. Nurse Education Today, 66(Julio), 103-109. https://doi.org/10.1016/j.nedt.2018.04.009

León, F. R. (2016). ¿Qué significa una tesis de investigación en psicología en el Perú?. Persona, (19), 151-166. https://dialnet.unirioja.es/descarga/articulo/6112762.pdf

Mayta-Tristán, P. (2016). Tesis en formato de artículo científico: oportunidad para incrementar la producción científica universitaria. Acta Médica Peruana, 33(2), 95-98. http://www.scielo.org.pe/scielo.php?script=sci_arttextypid=S1728$\underline{59172016000200001}$

Mayta-Tristán, P., Pereyra-Elías, R., \& Mejía, C. R. (2013). Producción científica de los miembros vitalicios de la Academia Nacional de Investigadores Médicos. Revista Peruana de Medicina Experimental y Salud Publica, 30(4), 714-728. http://www.scielo.org.pe/scielo.php?script=sci arttextypid=S172646342013000400033

Mejía, C. R., Valladares-Garrido, M. J., Luyo-Rivas, A., Valladares-Garrido, D., TalledoUlfe, L., Vilela-Estrada, M. A., \& Araujo Chumacero, M. M. (2015). Factores asociados al uso regular de fuentes de información en estudiantes de medicina de cuatro ciudades del Perú. Revista Peruana de Medicina Experimental y Salud Publica, $32(2)$, 230-236. http://www.scielo.org.pe/scielo.php?script=sci arttextypid=S172646342015000200003

Mejia, C. R., Caceres, O. J., Vera, C. A., Nizama-Vía, A., Curioso, W. H., \& MaytaTristán, P. (2014). Uso de fuentes de información en médicos recién graduados de Lima. Revista Peruana de Medicina Experimental y Salud Publica, 31(4), 716- 
720. http://www.scielo.org.pe/scielo.php?script=sci_arttext\&pid=S172646342014000400016

Mejia, C. R., Cáceres, O. J., Vera, C. A., Inga-Berrospi, F., \& Mayta-Tristán, P. (2016). Percepción y factores asociados a insatisfacción que los médicos recién graduados tienen de sus asesores de tesis, Lima-Perú. Revista Cubana de Educación Médica Superior, 30(4), 340-348. http://scielo.sld.cu/scielo.php?script=sci_arttext\&pid=S086421412016000400010

Mejia, C.R., \& Montero, C. (2018). Relación entre cantidad de alumnos de pregrado e investigadores registrados de universidades peruanas. Métodos y Recursos Educativos en Educación Médica, 20(s2),186-188. https://www.elsevier.es/esrevista-educacion-medica-71-pdf-S1575181318300688

MINEDU (2015). Ponte en carrera: observatorio de Educación y Empleo. Recuperado de https://www.ponteencarrera.pe/

Nordsteien, A., Horntvedt, M.T., \& Syse, J. (2017). Use of research in undergraduate nursing students' theses: A mixed methods study. Nurse Education Today, 56(setiembre), 23-28. https://doi.org/10.1016/j.nedt.2017.06.001

Obuku, E. A., Lavis, J. N., Kinengyere, A., Ssenono, R., Ocan, M., Mafigiri, D. K., \& Sewankambo, N. K. (2018). A systematic review on academic research productivity of postgraduate students in low-and middle-income countries. Health research policy and systems, 16(1), 86. https://doi.org/10.1186/s12961-018-03607

Oyola, A. E. (2015). El asesor de tesis (carta). Acta Médica Peruana, 32(2), 131-132. http://www.scielo.org.pe/scielo.php?script=sci arttext\&pid=S1728$\underline{59172015000200012}$

Pamo, O. G. (2005). Estado actual de las publicaciones periódicas científicas médicas del Perú. Revista Médica Herediana, 16(1), 65-73. http://www.scielo.org.pe/scielo.php?script=sci_arttext\&pid=S1018130X2005000100010

Pasarica, M., Bailey, M., \& Cendán, J. C. (2019). Increasing Students' Publication Productivity: Could Launching a University Scientific Journal be a Catalyst? Cureus, 11(1), e3953. https://doi.org/10.7759/cureus.3953

Pereyra-Elías, R., Huaccho-Rojas, J. J., Taype-Rondan, Á., Mejia, C. R., \& MaytaTristán, P. (2014). Publicación y factores asociados en docentes universitarios de investigación científica de escuelas de medicina del Perú. Revista Peruana de Medicina Experimental y Salud Pública, 31(3), 424-430. https://doi.org/10.17843/rpmesp.2014.313.76

Perez-Batres, L. A., Pisani, M. J., \& Doh, J. P. (2010). A perspective on international business scholarship: Is it regional or global? Multinational Business Review, 18(1), 73-88. https://doi.org/10.1108/1525383X201000004

Pulido-Medina, C., Hamon-Rugeles, D., López-Ramírez, E., Quimbayo-Cifuentes, A. F., \& Mejía, C. R. (2017). Publicación científica entre los directivos de la Asociación Científica de Estudiantes de Medicina de Colombia: características y factores asociados. Revista de la Facultad de Medicina, 65(4), 553-557. https://doi.org/10.15446/revfacmed.v65n4.60489

Rodríguez y. C., Cósar-Quiroz, J., Arredondo-Sierralta, T., \& Sihuay-Torres, K. (2018). Producción científica de tesis sustentadas y publicadas por estudiantes de Odontología. Educación $\quad$ Médica, $\quad 19(2), \quad 85-89$. https://doi.org/10.1016/j.edumed.2017.04.002 
Shukla, D., Tripathi, M., \& Devi, B. I. (2019). Conversion of Thesis to Peer-Reviewed Publication. Indian Journal of Neurosurgery, 8(2), 093-099. https://doi.org/10.1055/s-0039-1694959

Taype-Rondán, Á., Huaccho-Rojas J, Pereyra-Elías R, Mejia, C.R., \& Mayta-Tristán P. (2015). Características de los cursos de investigación en escuelas de medicina del Perú. Archivos de Medicina, 11(2), http://www.archivosdemedicina.com/abstract/caractersticas-de-los-cursosdeinvestigacin-en-escuelas-demedicina-del-per-5803.html

Taype-Rondán, Á., Carbajal-Castro, C., \& Arrunategui-Salas., G. (2015). Limitada publicación de tesis de pregrado en una facultad de medicina de Lima, Perú, 20002009. Anales de la Facultad de Medicina, 73(2), 153-157. https://revistasinvestigacion.unmsm.edu.pe/index.php/anales/article/view/858

Yagui, M., Espinoza, M., Caballero, P., Castilla, T., Garro, G. Yamaguchi, L. P., \& Cabezas, C. (2010). Avances y retos en la construcción del sistema nacional de investigación en salud en el Perú. Revista Peruana de Medicina Experimental y Salud Pública, 27(3), 387-397. http://www.scielo.org.pe/scielo.php?script=sci_arttext\&pid=S1726$\underline{46342010000300012}$

Wilson, K. (2002). Quality assurance issues for a PhD by published work: A case study. Quality Assurance in Education, 10(2), 71-78. https://doi.org/10.1108/09684880210423555

Zafra-Tanaka, J. H., \& Castillo, S. (2016). Barreras percibidas por los estudiantes de Medicina Humana para la titulación por tesis en la Universidad Nacional Mayor de San Marcos, Lima, Perú, 2015. Anales de la Facultad de Medicina, 77(2), 143146. https://doi.org/10.15381/anales.v77i2.11819 